INVESTIGACIÓN

\title{
FACTORES ASOCIADOS A LA DESERCIÓN ACADÉMICA EN LOS PROGRAMAS DE LAS ESCUELAS DE LA UNIVERSIDAD NACIONAL ABIERTA Y A DISTANCIA - UNAD- CCAV CARTAGENA*
}

\author{
FACTORS ASSOCIATED WITH THE DESERTION \\ OF STUDENTS FROM THE DIFFERENT SCHOOLS OF THE \\ "UNIVERSIDAD NACIONAL ABIERTA Y A DISTANCIA" UNAD \\ IN THE COMMUNITY CENTER OF VIRTUAL ATTENTION \\ (CCAV) OF CARTAGENA.
}

Julio César Sánchez-Sánchez ${ }^{*}$

Recibido: 7 de abril de 2018

Evaluado: 19 de mayo de 2018

Aprobado: 5 de junio de 2018

\section{RESUMEN}

La presente investigación tiene como objetivo identificar los diferentes factores asociados a la deserción de estudiantes de las diferentes escuelas de la Universidad Nacional Abierta y a Distancia UNAD en el Centro Comunitario de Atención Virtual (CCAV) de Cartagena. Es empírico-analítica, de tipo ex post facto con cuantitativo - descriptivo. Del total de la población de desertados en el periodo de estudio se tomó una muestra de 210 estudiantes.

* Producto resultado de un proyecto de investigación financiado por la Universidad Nacional Abierta y a Distancia UNAD. Artículo de investigación científica

** Economista Universidad Tecnológica de Bolívar, Especialista en Técnicas de Investigación en Ciencias Sociales Universidad de Cartagena, Magister en Administración Instituto Tecnológico de Monterrey, Docente Asistente Universidad Nacional Abierta y a Distancia UNAD, mail jcsanchezsa@gmail.com, julio.sanchez@unad.edu.co, móvil 3145657252, Transversal 45 \# 44A -221 barrio Paraguay Cartagena de Indias Colombia. https://orcid.org/0000-0003-4836-1473 
Teniendo como base teórica dos grandes marcos interpretativos, uno que enfatiza en agentes exteriores como situación socioeconómica, condiciones laborales, horas de dedicación al estudio y contexto familiar; y el otro que tiene en cuenta los agentes internos como problemas motivacionales, personales, desempeño, bajo rendimiento, mala conducta y edad, y se analizan factores individuales, familiares, relacionados con la institución educativa, y lo regional y contextual, encontrándose que el mayor peso lo tienen en su orden, lo económico, los métodos inadecuados de estudio, la adaptación al sistema, el tipo de trabajo en que se desempeña el estudiante y las bases inadecuadas de formación con la cual llegan.

\begin{abstract}
The objective of this research is to identify the different factors associated with the desertion of students from the different schools of The "Universidad Nacional Abierta Y a Distancia" UNAD in the Community Center of Virtual Attention (CCAV) of Cartagena. It is empirical-analytical, ex post facto type with quantitative - descriptive. Of the total defected population in the study period, a sample of 210 students was taken.
\end{abstract}

Getting the theoretical basis two great interpretive frameworks, one that emphasizes on external agents such as socioeconomic situation, working conditions, hours of dedication to study and family context; and the other one, that takes into account internal agents as motivational problems, personal, performance, poor performance, misconduct and age, and individual family, related to the school, and regional and contextual factors are analyzed and found to be the biggest weight they have in their order, the economic, the inadequate methods of study, the adaptation to the system, the type of job in which the student performs and the inadequate bases of training with which they arrive.

Palabras clave: Deserción, Educación a distancia, Educación virtual, Factores de deserción, UNAD Keywords: Desertion, Distance education, Desertion factor, Virtual Education 


\section{INTRODUCCIÓN}

La deserción estudiantil es uno de los problemas que más afecta tanto a las instituciones educativas como a los mismos estudiantes y a la sociedad en general. A las instituciones educativas en cuanto deben realizar esfuerzos significativos paracaptar un número adecuado de estudiantes que les aseguren un funcionamiento de acuerdo con unos planes preestablecidos, pero que al no lograr que estos se mantengan en el proceso formativo ven incrementados sus costos de operación; a los estudiantes que no logran permanecer en el proceso les genera inseguridad y desconcierto sobre un mejor futuro; y a la sociedad en general le produce un rezago en la formación de un recurso humano que le permita procesos de desarrollo adecuados.

Para el caso de Colombia, existen diferentes sistemas y organizaciones que buscan estudiar, analizar, y mantener información con respecto a este fenómeno tales como el Sistema para la Prevención y Análisis de la Deserción en las Instituciones de Educación Superior (SPADIES), el Sistema Nacional de Información de la Educación Superior (SNIES), el Sistema de Aseguramiento de la Calidad de Educación Superior (SACES), y por el Instituto Colombiano para el Fomento de la Educación Superior (ICFES).

Teniendo como marco lo anterior, se pretende analizar como es el comportamiento de la deserción en el CCAV Cartagena de la UNAD y cuáles son los factores que inciden sobre ella.

\section{DESCRIPCIÓN DEL PROBLEMA}

Los retos actuales de desarrollo social, económico y productivo del país demandan una educación de alta calidad que responda con pertinencia a las necesidades del entorno nacional, regional y local con perspectiva globales y que doten a los estudiantes de las competencias que requieren como ciudadanos del siglo XXI (De Witte \& Rogge, 2013). La calidad debe darse mediante una sólida formación de las competencias básicas, sobre las cuales se construye el conocimiento científico-tecnológico, y unas competencias ciudadanas que aseguren altos estándares éticos (Lugo, 2013). Además, debe lograr la pertinencia para que los jóvenes se formen en áreas relevantes para el desarrollo, de tal forma que puedan encontrar oportunidades para construir un proyecto profesional que los incentive a educarse a lo largo de su vida y los empodere para insertarse laboralmente de forma inmediata o en el mediano plazo (Espinoza, et al., 2012; Rinne \& Järvinen, 2011).

En relación con la situación descrita, es importante observar la preocupación cada vez más acelerada, acerca del deterioro de la educación superior en el ámbito nacional, reflejado en las altas tasas de deserción académica, especialmente en las Facultades como las de 
Ingeniería y afınes. Según el estudio "Análisis de determinantes de la deserción en la educación superior Colombiana con base en el SPADIES (2008)", realizado por el Ministerio de Educación Nacional de Colombia, en los últimos años en Colombia se ha venido incrementando el nivel de deserción en las Instituciones de Educación Superior públicas y privadas, esto obedece a que los jóvenes no encuentran un espacio donde se fomente la reflexión, la formación integral y permanente en el ámbito académico, científico y humanístico que le permita Identificarse con su carrera y perseguir su vocación; por el contrario, la participación del estudiantado, en la mayoría de los casos, se ha reducido a cumplir con unos requisitos para obtener un cartón en el menor tiempo posible.

Al estudiar esta realidad se han determinado causas que llevan a la deserción, académica, pero que según Baquerizo et al., (2014), son diferentes según el contexto donde se analicen; entre estas podemos destacar las de tipo económico, familiar, psicológico, personal y propias de la Institución académica, lo cual retarda el inicio de la vida profesional, afecta las Instituciones Universitarias ya que disminuyen sus recursos monetarios, contribuye con el empobrecimiento intelectual y resta la oportunidad de estudiar a otras personas para formarse integral, social e interculturalmente (Espinoza, et al., 2012; Barragán, 2011).

\section{FORMULACIÓN DEL PROBLEMA}

Descritos los problemas anteriores, se plantea entonces el siguiente interrogante. ¿Cuáles son los factores más relevantes que ocasionan los fenómenos de la deserción académica en las Escuelas de la de la Universidad Nacional Abierta y a Distancia UNAD CCAV Cartagena?

\section{MARCO TEÓRICO}

La deserción y la repitencia son fenómenos que además de estar íntimamente relacionados entre sí tienen una relevancia fundamental en educación pues son, en la mayoría de los casos, causa principal del fracaso educativo (Espínola, \& Claro, 2010). Comprender el fenómeno de la deserción, implica considerar que, en la realidad, el proceso de abandono de los estudios es el resultado de la interacción de múltiples factores y cuya complejidad puede resumirse en la existencia de causas de "expulsión" del sistema -inadecuación de la oferta educativa-, y de "atracción" de otros campos sociales o del ámbito laboral (UNICEF, 2012). Para organizar el tema, podrían agruparse los factores causantes de la deserción en dos grandes marcos interpretativos:

1. El que enfatiza en los agentes exteriores que incluye la situación socioeconómica, las condiciones laborales, y por ende las horas de dedicación al estudio, además del contexto familiar. 
2. Y, por otro lado, los factores internos que abarcan los problemas motivacionales, personales y psico-afectivos, sentimientos de frustración, desorientación vocacional, baja autoestima y la adaptación al medio-; problemas de desempeño, como el bajo rendimiento, la mala conducta y problemas asociados a la edad (Cabus \& DeWitte, 2011; UNICEF, 2012), o bien vinculados con la idoneidad, autoritarismo y metodología docente.

Inevitablemente también es necesario considerar los aspectos que hacen al entorno externo del individuo como la estabilidad política del país, la superpoblación de las aulas de clase; la carencia de métodos modernos o de espacios adecuados para la enseñanza (Castro et al. 2010). Así como los que se derivan del propio sistema: como la rigidez de los programas de estudios, su desvinculación y desarticulación con la realidad social y el mercado de trabajo, siendo todos estos elementos, parte de la misma problemática (Cratty, 2012). En este sentido, la propia estructura, los actores que forman parte de ella y el tipo de relaciones que se generan, serían los responsables directos de los elementos expulsores (De Witte, \& Rogge, 2013). La deserción, por tanto, involucra factores económicos, culturales, sociales o circunstanciales, que hacen que el estudiante no continúe con sus estudios, y en estos últimos tiempos, se ha constituido en un fenómeno colectivo o incluso masivo asociado, indefectiblemente, con la deficiencia del sistema educativo (Espínola, \& Claro, 2010; De Witte, \& Rogge, 2013). Desde el punto de vista metodológico la medición de la deserción ostenta varios inconvenientes, primero, no puede establecerse de una manera absoluta, pues un estudiante puede abandonar en un determinado curso lectivo y reincorporarse después (Fall, \& Roberts, 2012). A la vez, un alumno puede concluir el curso, pero no inscribirse al siguiente año lectivo. Por tanto, pueden distinguirse dos tipos de desertores:

1. Los que ingresan y abandonan sin terminar el curso.

2. Los que terminan el curso, pero no se inscriben a los cursos superiores.

El sistema educativo puede detectar más fácilmente a los primeros calculando el porcentaje de deserción como la razón entre matrícula a inicio y matrícula al finalizar el año (UNICEF, 2012). Pero el sistema educativo no cuenta en la mayoría de los casos con los mecanismos apropiados para hacer un seguimiento de aquellos estudiantes que abandonan sus estudios, tampoco para indagar en sus dificultades, ofrecerles apoyo profesional, material o para reinsertarlos 
en el sistema (Román, 2013). Por su parte la repitencia, es entendida como la acción de cursar reiterativamente una actividad académica, sea por mal rendimiento del estudiante o por causas ajenas al ámbito educativo (Vera-Noriega et al. 2012). Se llama rezago, a la prolongación de los estudios por sobre lo formalmente establecido para cada curso, y comúnmente, se acepta este atraso educativo, como un indicador de la repitencia (Villalonga, 2011).

\section{METODOLOGÍA}

- Tipo de investigación: empírico-analítica, de tipo ex post facto, ya que según Bisquerra (1989), estas se plantean "cuando el fenómeno ya ha sucedido, y generalmente se trata de una búsqueda de las causas que los han producido".

- Enfoque: cuantitativo - descriptivo, ya que se pretende mostrar índices de deserción académica en los programas de las escuelas de la Universidad Nacional Abierta y a Distancia -UNAD- CCAV Cartagena, así como identificar y describir los factores que ocasionan estas problemáticas.

- Población: Estudiantes matriculados en el periodo de 2008 al 2014, este trabajo es de corte transversal.
- Muestra: estudiantes se seleccionaron según la ecuación:

$$
n=\frac{N Z^{2} p q}{i^{2}(n-1)+Z^{2} p q}
$$

\section{RESULTADOS}

Para el periodo de estudio se analizan Veinte Mil Cuatrocientos Cuarenta y Seis (20.446) registros de matrícula correspondientes a los alumnos nuevos y antiguos matriculados tanto a nivel de Pregrado (Profesional y Tecnológico) como en Posgrado en los diferentes periodos comprendidos entre el 2008-1 y el 2014-2 en las diferentes escuelas, estandarizando dos (2) periodos por año, para el periodo 1 se toman los periodos de 16 semanas y los periodos de 8 semanas que se inician entre Enero y Junio y para el periodo 2 se toman los periodos de 16 semanas y los periodos de 8 semanas que se inician entre Julio y Noviembre.

Del total de matrículas analizadas, 19.945 (97.5\%) corresponden a programas de Pregrado y $501(2,5 \%)$ a programas de posgrado, y del nivel de pregrado, 12.232 matrículas (61.3\%) corresponden a programas profesionales y 7.713 matrículas (38.7\%) corresponden a programas tecnológicos (Tabla 1). 


\section{Estrategia Organizacional}

Tabla 1. Matrículas totales en el periodo de estudio.

\begin{tabular}{|c|c|c|c|c|c|}
\hline Periodo & $\begin{array}{c}\text { Total } \\
\text { Matrículas }\end{array}$ & Pregrado & Profesional & Tecnologías & Posgrado \\
\hline $\mathbf{2 0 0 8 - 1}$ & 971 & 966 & 734 & 232 & 5 \\
\hline $\mathbf{2 0 0 8 - 2}$ & 1.371 & 1.347 & 703 & 644 & 24 \\
\hline $\mathbf{2 0 0 9 - 1}$ & 2.184 & 2.155 & 885 & 1.270 & 29 \\
\hline $\mathbf{2 0 0 9 - 2}$ & 1.087 & 1.057 & 680 & 377 & 30 \\
\hline $\mathbf{2 0 1 0 - 1}$ & 1.471 & 1.444 & 736 & 708 & 27 \\
\hline $\mathbf{2 0 1 0 - 2}$ & 1.574 & 1.539 & 793 & 746 & 35 \\
\hline $\mathbf{2 0 1 1 - 1}$ & 1.479 & 1.460 & 949 & 511 & 19 \\
\hline $\mathbf{2 0 1 1 - 2}$ & 1.967 & 1.925 & 1.148 & 777 & 42 \\
\hline $\mathbf{2 0 1 2 - 1}$ & 1.854 & 1.819 & 1.192 & 627 & 35 \\
\hline $\mathbf{2 0 1 2 - 2}$ & 1.540 & 1.508 & 961 & 547 & 32 \\
\hline $\mathbf{2 0 1 3 - 1}$ & 1.382 & 1.338 & 910 & 428 & 44 \\
\hline $\mathbf{2 0 1 3 - 2}$ & 1.408 & 1.343 & 962 & 381 & 65 \\
\hline $\mathbf{2 0 1 4 - 1}$ & 964 & 902 & 700 & 202 & 62 \\
\hline $\mathbf{2 0 1 4 - 2}$ & 1.194 & 1.142 & 879 & 263 & 52 \\
\hline Totales & 20.446 & 19.945 & 12.232 & 7.713 & 501 \\
\hline Promedio & $\mathbf{1 . 4 6 0}$ & $\mathbf{1 . 4 2 5}$ & $\mathbf{8 7 4}$ & $\mathbf{5 5 1}$ & $\mathbf{3 6}$ \\
\hline
\end{tabular}

Fuente: Elaborado por el autor con los datos obtenidos de R y C local

Del total de matrículas en el periodo de estudio, 5.101 fueron nuevas (24.9\%) y 15.345 fueron antiguas (75.1\%) (Tabla 2) con un promedio de 364 matrículas nuevas por periodo y 1.096 matrículas antiguas por periodo. 
Factores asociados a la deserción académica en los programas de las escuelas de la Universidad Nacional Abierta y a Distancia - UNAD- CCAV Cartagena

Tabla 2. Tipos de matrículas en el periodo analizado

\begin{tabular}{|c|c|c|}
\hline Periodo & Matrículas Nuevas & Matrículas Antiguas \\
\hline $\mathbf{2 0 0 8 - 1}$ & 257 & 714 \\
\hline $\mathbf{2 0 0 8 - 2}$ & 647 & 724 \\
\hline $\mathbf{2 0 0 9 - 1}$ & 1.155 & 1.029 \\
\hline $\mathbf{2 0 0 9 - 2}$ & 96 & 991 \\
\hline $\mathbf{2 0 1 0 - 1}$ & 432 & 1.039 \\
\hline $\mathbf{2 0 1 0 - 2}$ & 460 & 1.114 \\
\hline $\mathbf{2 0 1 1 - 1}$ & 198 & 1.281 \\
\hline $\mathbf{2 0 1 1 - 2}$ & 306 & 1.661 \\
\hline $\mathbf{2 0 1 2 - 1}$ & 197 & 1.657 \\
\hline $\mathbf{2 0 1 2 - 2}$ & 458 & 1.082 \\
\hline $\mathbf{2 0 1 3 - 1}$ & 201 & 1.181 \\
\hline $\mathbf{2 0 1 3 - 2}$ & 248 & 1.160 \\
\hline $\mathbf{2 0 1 4 - 1}$ & 240 & 724 \\
\hline $\mathbf{2 0 1 4 - 2}$ & 206 & 988 \\
\hline Totales & $\mathbf{5 . 1 0 1}$ & $\mathbf{1 5 . 3 4 5}$ \\
\hline Promedio & $\mathbf{3 6 4}$ & $\mathbf{1 . 0 9 6}$ \\
\hline
\end{tabular}

Fuente: Elaborado por el autor con los datos obtenidos de R y C local.

En cuanto a la participación por escuelas (Tabla 3), en orden de participación se encuentra a la escuela ECBTI con el 30.34\% (6.204 matriculas), ECSAH con el 23.14\% (4.731 matrículas), ECACEN con el 23.03\% (4.708 matrículas), ECADU con el 15.7\% (3.211 matrículas), ECISALUD con el $5.11 \%$ (1.044 matrículas) y ECAPMA con el 2.68\% (548 matrículas). Como se puede observar, tres escuelas, ECBTI, ECACEN, ECSAH aportan el $76.51 \%$ de las matrículas totales del periodo de estudio.

Tabla 3. Participación por escuelas en el total de matriculas

\begin{tabular}{|l|l|}
\hline \multicolumn{1}{|c|}{ ESCUELA } & \multicolumn{1}{c|}{ CANTIDAD } \\
\hline CIENCIAS BÁSICAS TECNOLOGÍA E INGENIERÍA - ECBTI & 6.204 \\
\hline CIENCIAS SOCIALES, ARTES Y HUMANIDADES - ECSAH & 4.731 \\
\hline CIENCIAS ADTIVAS, CONTABLES, ECONOMICAS Y DE NEGOCIOS - ECACEN & 4.708 \\
\hline CIENCIAS DE LA EDUCACIÓN - ECEDU & 3.211 \\
\hline CIENCIAS DE LA SALUD - ECISALUD & 1.044 \\
\hline CIENCIAS AGRICOLAS, PÉCUARIAS Y DEL MEDIO AMBIENTE - ECAPMA & 548 \\
\hline TOTAL & 20.446 \\
\hline
\end{tabular}

Fuente: Elaborado por el autor con los datos obtenidos de R y C local 


\section{DESERCIÓN:}

Durante el periodo de estudio se encuentra qué de las 5.101 matrículas nuevas, abandonaron o desertaron del proceso académico 1.163 lo que representa un 22.8\% (Tabla 4), los periodos que presentan mayor deserción son 2012-1 con el $47.7 \%$, seguido del 2013-1 con el $41.8 \%$, el 2009 2 con el $41.7 \%$ y los demás periodos están por debajo del $40 \%$

Tabla 4. Deserción por periodo.

\begin{tabular}{|l|l|l|}
\hline \multicolumn{1}{|c|}{ PERIODO } & \multicolumn{1}{c|}{ Desertaron } & \multicolumn{1}{c|}{$\%$} \\
\hline $\mathbf{2 0 0 8 - 1}$ & 88 & 34.2 \\
\hline $\mathbf{2 0 0 8 - 2}$ & 103 & 15.9 \\
\hline $\mathbf{2 0 0 9 - 1}$ & 155 & 13.4 \\
\hline $\mathbf{2 0 0 9 - 2}$ & 40 & 41.7 \\
\hline $\mathbf{2 0 1 0 - 1}$ & 88 & 20.4 \\
\hline $\mathbf{2 0 1 0 - 2}$ & 87 & 18.9 \\
\hline $\mathbf{2 0 1 1 - 1}$ & 40 & 20.2 \\
\hline $\mathbf{2 0 1 1 - 2}$ & 78 & 25.5 \\
\hline $\mathbf{2 0 1 2 - 1}$ & 94 & 47.7 \\
\hline $\mathbf{2 0 1 2 - 2}$ & 139 & 30.3 \\
\hline $\mathbf{2 0 1 3 - 1}$ & 84 & 41.8 \\
\hline $\mathbf{2 0 1 3 - 2}$ & 74 & 29.8 \\
\hline $\mathbf{2 0 1 4 - 1}$ & 79 & 32.9 \\
\hline $\mathbf{2 0 1 4 - 2}$ & 14 & 6.8 \\
\hline Totales & 1.163 & 22.8 \\
\hline
\end{tabular}

Fuente: Elaborada por el autor con los datos obtenidos de RyC

Analizando la deserción por escuela durante el periodo de estudio, y con relación al número de matriculados (Tabla 5), se encuentra que las mayores deserciones se presentan en la Escuela de Ciencias de la Educación ECEDU con el $42.7 \%$, seguida de la Escuela de Ciencias, Artes y Humanidades ECSAH con el 37.6\%, la Escuela de Ciencias de la Salud con el $9.8 \%$ 
Tabla 5. Deserción Escuela.

\begin{tabular}{|c|c|c|c|c|c|c|c|c|c|c|c|c|}
\hline 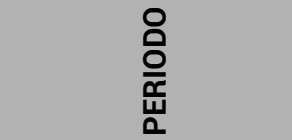 & $\begin{array}{l}\bar{F} \\
\text { 岀 }\end{array}$ & ๙ீ & $\begin{array}{l}\sum_{0}^{\nwarrow} \\
\text { 充 } \\
\text { }\end{array}$ & ๙ீ & $\begin{array}{l}\text { Zu } \\
\text { U্র }\end{array}$ & ภீ & エ্ড & ১ீ & 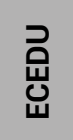 & ๙ீ & હ & ஃீ \\
\hline 2008-1 & 34 & 50.0 & 3 & 42.9 & 18 & 29.0 & 26 & 38.2 & 7 & 46.7 & 5 & 13.5 \\
\hline 2008-2 & 31 & 11.0 & 1 & 4.5 & 13 & 7.0 & 11 & 39.3 & 47 & 45.6 & 6 & 23.1 \\
\hline 2009-1 & 25 & 5.2 & 3 & 12.5 & 17 & 4.9 & 14 & 38.9 & 96 & 47.8 & 3 & 4.8 \\
\hline $2009-2$ & 18 & 58.1 & 6 & 75.0 & 6 & 25.0 & 9 & 36.0 & 1 & 16.7 & 0 & 0.0 \\
\hline 2010-1 & 38 & 22.6 & 4 & 25.0 & 9 & 8.2 & 16 & 40.0 & 21 & 48.8 & 4 & 7.3 \\
\hline 2010-2 & 24 & 14.3 & 1 & 8.3 & 4 & 3.7 & 19 & 40.4 & 39 & 39.4 & 3 & 11.1 \\
\hline 2011-1 & 18 & 23.1 & 1 & 16.7 & 2 & 3.5 & 8 & 29.6 & 11 & 45.8 & 0 & 0.0 \\
\hline 2011-2 & 23 & 24.7 & 1 & 14.3 & 5 & 7.4 & 23 & 45.1 & 26 & 37.7 & 1 & 5.6 \\
\hline 2012-1 & 35 & 54.7 & 3 & 50.0 & 13 & 41.9 & 36 & 47.4 & 7 & 46.7 & 0 & 0.0 \\
\hline 2012-2 & 20 & 18.9 & 2 & 25.0 & 10 & 11.8 & 72 & 45.6 & 35 & 42.7 & 2 & 10.5 \\
\hline 2013-1 & 23 & 65.7 & 3 & 60.0 & 14 & 25.9 & 32 & 43.2 & 12 & 42.9 & 1 & 20.0 \\
\hline 2013-2 & 27 & 43.5 & 1 & 25.0 & 9 & 16.7 & 21 & 24.7 & 16 & 47.1 & 2 & 22.2 \\
\hline 2014-1 & 20 & 35.1 & 4 & 21.1 & 19 & 30.2 & 28 & 37.8 & 8 & 32.0 & 0 & 0.0 \\
\hline 2014-2 & 7 & 16.3 & 0 & 0.0 & 2 & 3.2 & 4 & 6.8 & 1 & 4.5 & 0 & 0.0 \\
\hline Totales y Promedio & 343 & 19.7 & 33 & 20.5 & 141 & 10.8 & 319 & 37.6 & 327 & 42.7 & 27 & 9.8 \\
\hline
\end{tabular}

Fuente: Elaborada por el autor con los datos obtenidos de RyC

\section{RAZONES DE DESERCIÓN}

Sometida la información recolectada al procesamiento mediante la utilización del software SPSS V22, se logra determinar el peso relativo de cada una de las razones, (Tabla 6) y se obtiene que fundamentalmente la deserción obedece a factores económicos con un peso del $21.73 \%$, los métodos inadecuados de estudio con el $11.14 \%$, la adaptación al sistema con el $10.25 \%$, el tipo de trabajo que realiza el estudiante con el $9.02 \%$, las bases inadecuadas de formación con el $7.61 \%$, y los modelos pedagógicos diferentes a los acostumbrados como las razones más significativas 
Tabla 6. Peso relativo de cada razón de abandono.

\begin{tabular}{|c|c|}
\hline Razón de abandono & Peso \% \\
\hline Factores Económicos. & $21,73 \%$ \\
\hline Métodos Inadecuados de Estudios. & $11,14 \%$ \\
\hline Adaptación al sistema & $10,25 \%$ \\
\hline Tipo de trabajo & $9,02 \%$ \\
\hline Bases inadecuadas de formación. & $7,61 \%$ \\
\hline Modelos pedagógicos diferentes a los acostumbrados. & $7,40 \%$ \\
\hline Programas curriculares rígidos, de alta intensidad temática. & $4,25 \%$ \\
\hline La carrera no Satisfizo sus Expectativas & $3,70 \%$ \\
\hline Inadecuada Orientación Profesional. & $3,53 \%$ \\
\hline Viajes por trabajo & $3,36 \%$ \\
\hline Perdió varias veces la(s) misma(s) asignaturas. & $2,81 \%$ \\
\hline Embarazo. & $2,81 \%$ \\
\hline Problemas emocionales y afectivos & $2,64 \%$ \\
\hline Problemas de salud. & $2,47 \%$ \\
\hline Ambientes familiares conflictivos y violentos. & $2,30 \%$ \\
\hline Evaluaciones extenuantes y avasalladoras. & $2,13 \%$ \\
\hline Aptitud vocacional & $1,95 \%$ \\
\hline Disciplinarias. & $0,89 \%$ \\
\hline
\end{tabular}

Fuente: Datos obtenidos por el autor del software SPSS aplicado a la encuesta. 


\section{CAUSA E IMPACTO DE LA RAZÓN DE DESERCIÓN O ABANDONO}

\begin{tabular}{|c|c|c|}
\hline TIPO & CAUSA & IMPACTO \\
\hline ECONÓMICO & $\begin{array}{l}\text { Ingresos Menores de } 3 \mathrm{SM} \\
\text { Monto de la matricula } \\
\rightarrow \text { Tener más de } 2 \text { hijos } \\
\text { Tener más de } 3 \text { personas a cargo } \\
\text { Matricula con beca }\end{array}$ & $\begin{array}{l}\rightarrow \text { Alto } \\
\rightarrow \text { Alto } \\
\rightarrow \text { Alto } \\
\rightarrow \text { Alto } \\
\rightarrow \text { Medio }\end{array}$ \\
\hline $\begin{array}{l}\text { METODOS } \\
\text { INADECUADOS } \\
\text { DE ESTUDIO }\end{array}$ & $\begin{array}{l}\text { Desconoce método para trabajo independiente } \\
\text { No asigna el tiempo requerido para las actividades } \\
\rightarrow \text { Poca experiencia en metodolodologia a distancia } \\
\text { Cree en mayor facilidad dela metodologia a distancia } \\
\text { Dificultad para el trabajo grupal }\end{array}$ & $\begin{array}{l}\rightarrow \text { Alto } \\
\rightarrow \text { Alto } \\
\rightarrow \text { Alto } \\
\rightarrow \text { Medio } \\
\rightarrow \text { Medio }\end{array}$ \\
\hline $\begin{array}{l}\text { ADAPTACIÓN } \\
\text { ALSISTEMA }\end{array}$ & $\begin{array}{l}\text { Poca claridad sobre el sistema utilizado por la UNAD al ingresar } \\
\text { Muchas actividades con cierre al mismo tiempo } \\
\text { Poca claridad de las guias de actividades } \\
\text { Sensación de soledad en el campus } \\
\text { Pocas destrezas en manejos de herramientas tecnológicas }\end{array}$ & $\begin{array}{l}\rightarrow \text { Alto } \\
\rightarrow \text { Alto } \\
\rightarrow \text { Alto } \\
\rightarrow \text { Medio } \\
\rightarrow \text { Medio }\end{array}$ \\
\hline $\begin{array}{c}\text { TIPO DE } \\
\text { TRABAJO }\end{array}$ & $\begin{array}{l}\text { Trabajo con imposibilidad de conectividad } \\
\text { Trabajo con jornadas extenuantes } \\
\text { Trabajos en luagres fuera de cobertura tecnológica } \\
\text { Trabajo en actividades de mucho desgaste físico } \\
\text { Trabajo que requiere largos deplazamientos }\end{array}$ & $\begin{array}{l}\rightarrow \text { Alto } \\
\rightarrow \text { Medio } \\
\rightarrow \text { Medio } \\
\rightarrow \text { Medio } \\
\rightarrow \text { Bajo }\end{array}$ \\
\hline $\begin{array}{l}\text { BASES } \\
\text { INADECUADAS } \\
\text { DE FORMACIÓN }\end{array}$ & $\begin{array}{l}\text { Periodos largos sin estudiar } \\
\text { Desconocimiento de la temática del programa matriculado } \\
\rightarrow \text { Poca actualización al momento de matricula } \\
\text { Matricular cursos que requerian prerrequisitos } \\
\text { No recurrir a apoyo institucional }\end{array}$ & $\begin{array}{l}\rightarrow \text { Medio } \\
\rightarrow \text { Medio } \\
\rightarrow \text { Medio } \\
\rightarrow \text { Bajo } \\
\rightarrow \text { Bajo }\end{array}$ \\
\hline
\end{tabular}

\section{RECOMENDACIONES}

En cuanto a los factores económicos, se debe buscar dirigir la mirada hacia futuros estudiantes con ingresos superiores a los 3 salarios mínimos que puedan asumir el pago de la matrícula prevista, dado que por los costos institucionales incurridos en el desarrollo de la actividad no es posible su disminución. En el periodo de estudio el número de matrículas subsidiadas fue considerable, por lo que se hace necesario buscar estudiantes que se matriculen con recursos propios y las becas o subsidios sean de carácter marginal. 
Respecto de los métodos inadecuados de estudio, los impactos más significativos se encuentran en el desconocimiento del método de estudio, la no asignación del tiempo requerido para las actividades, la poca experiencia en la metodología a distancia, la creencia de que la metodología a distancia es algo fácil y la dificultad para el trabajo grupal. Para mejorar en estos aspectos se recomienda implementar una capacitación específicamente y unificada que trate sobre estos temas las dos primeras semanas con una valoración igual a los 25 puntos que tiene cada actividad inicial de todos y cada uno de los cursos matriculados, de esta manera se asegura que los estudiantes conozcan y se familiaricen con la metodología aprendizaje autónomo, colaborativo y significativo.

Sobre la adaptación al sistema, se encuentra que el mayor impacto se encuentra en la poca claridad que tiene el estudiante nuevo sobre lo que es el sistema de aprendizaje virtual, situación que ayuda a corregir la capacitación específica de la cual se trata en el punto anterior. En cuanto al cierre de muchas actividades simultáneamente, se podría explorar la posibilidad de que se desarrolle un solo curso durante cada 4 semanas sólo para los estudiantes de primera matrícula a fin de que centren sus esfuerzos y no se sientan avasallados por la presentación de actividades; a partir de la segunda matrícula, permitir que los estudiantes desarrollen simultáneamente hasta dos cursos cada 4 semanas puesto que ya han internalizado de mejor manera el sistema de estudio. Otra causa de alto impacto que manifestaron los estudiantes que desertaron es que encontraron poca claridad en las guías de actividades las cuales presentan un abundante volumen de información que hace poco amigable su abordaje, para ello se recomienda hacer una revisión de cada una de ellas y simplificarlas sin sacrificar la exigencia en cada una de las actividades.

Frente al tipo de trabajo que realizan los estudiantes, tiene un impacto medio en la deserción y es muy poco lo que institucionalmente se puede hacer, salvo, conocer de manera más cercana la situación laboral de cada uno y buscar ofrecerles las oportunidades para que cumplan con sus obligaciones cuando así se requiera.

En cuanto a que muchos consideraron que tenían bases inadecuadas de formación al momento de ingresar a cursar algún programa, se recomienda identificar este tipo de falencias antes de permitirles matricular los cursos regulares, y ofrecerles la oportunidad de realizar algunas jornadas de actualización que sería necesarias para asegurar un normal desempeño académico del estudiante y así contribuir a disminuir la posibilidad de deserción por este motivo. 


\section{REFERENTES BIBLIOGRÁFICOS}

Abar, B., Abar, C.C., Lippold, M., Powers, C.J., \&Manning A.E. (2012). Associations between reasons to attend and late high school dropout. Learning and Individual Differences, 22(6), 856-86.

Alcántara J. "Como educar la autoestima". Métodos, Estrategias, y Actividades. CEAC. (1998).

Andrei, T., Teodorescu, D., Oancea, B. (2010). Characteristics of highereducation in Romania during transition, Procedia - Social and Behavioral Sciences, 2 (2), 3417-3421.

Baquerizo, C. y otros. La repitencia en las instituciones de Educación Superior: algunas experiencias investigativas en el Ecuador." Universidad y Sociedad: 6. (1), 1-10 (2014).

Barragán M. La carga horaria excesiva, factor del bajo rendimiento en Ingeniería Civil: Un estudio de Caso. Revista Tecnología, Investigación y Docencia, 6, 67-73. (2011).

Bjerk, D. (2012). Re-examining the impact of dropping out on criminal and labor outcomes in early adult hood. Economics of Education Review, 31(1), 110-122.
Cabus, S.J. \& De Witte, K. (2011). Does school time matter?-on the impact of compulsory education age on school dropout. Economics of Education Review, 30, 1384- 1398.

Castaño, E., S .Gallón y J. Vásquez. Análisis de los factores asociados a la deserción estudiantil en la educación superior: un estudio de caso. Revista de Educación: 1 (2), 255-280, (2008).

Castro, M., Ruiz, L.S., León, A.T., Fonseca, H., Díaz, M. \& Umaña, W. (2010). Factores académicos en la transición de la primaria a la secundaria: motivación, rendimiento académico y disciplina. Actualidades Investigativas en Educación, 10(3), 1-29.

Cerpa, F., P. González y S. Cantillo. Análisis comparativo entre las características más relevantes de deserción estudiantil en el programa de Ingeniería Industrial de la Universidad Autónoma del Caribe. Escenarios: 12(1), 96-104, (2014).

Cratty, D. (2012). Potential for significant reductions in dropout rates: Analysis of an entire 3rd grade state cohort. Economics of Education Review, 31(5), 644-662. 
De Witte, K. y N. Rogge, Dropout from secondary education: All's well that begins well. European Journal of Education: 47(4), 1-20 (2013).

Espínola, V., \& Claro, J. (2010). Estrategias de prevención de la deserción en la Educación Secundaria: perspectiva latinoamericana. Revista de Educación, 1(2), 257-280.

Espinoza, O. y otros tres autores, Factores familiares asociados a la deserción estudiantil en Chile. Revista de Ciencias Sociales: 18 (1), 136150 (2012).

Espinoza, O., Castillo, D., González, L.E. \& Loyola, J. (2012). Factores familiares asociados a la deserción escolar en Chile. Revista de Ciencias Sociales, 18(1), 136-150.

Fall, A. M., \& Roberts, G. (2012). High school dropouts: Interactions between social context, self-perceptions, school engagement, and student dropout. Journal of Adolescence, 35(1), 787-798.

Fleisch, B., Shindler, J., Perry, H., 2012. Whoisout of school? Evidence from the statistics South Africa community survey. International Journal of Educational Development, 32(1), 529- 536.
García, S., Fernández, C., \& Sánchez, F. (2010). deserción y Repetición en los primeros grados de la básica primaria: factores de riesgo y alternativas de política pública. Bogotá, Colombia: Gente nueva editorial Ltda.

González, L. Estudio sobre la repitencia y deserción en la educación superior Chilena. Digital Observatory for higher education in Latin America and The Caribbean, IESALC-UNESCO, Santiago-Chile (2005).

Gvirtz, S. \&Oría, A.I. (2010). Evaluar el rendimiento interno y académico: un desafío para la macro y el micro político. Lecciones a partir de un estudio de caso. Revista Iberoamericana de Evaluación Educativa, 3(2), 127-144.

Hanafi, S., Chaaban, J., Seyfert, K., 2012. Social exclusion of Palestinian refugees in Lebanon: reflections on the mechanisms that cement the persistent poverty. Refugee Survey Quarterly, 31(2), 1-20.

Lucio, R., Hunt, E., \& Bornovalova, M. (2012). Identifying the necessary and sufficient number of risk factors for predicting academic failure. Development al Psychology, 48, 422-428. 
Lugo, B. (2013). La deserción estudiantil: ¿realmente es un problema social? Revista de Postgrado FACE-UC, 7(12), 289-309.

Lugo, B. y otros cuatro autores. La deserción estudiantil: ¿realmente es un problema social? Revista de Postgrado FACE-UC: 7(12), 289-309 (2013).

Markussen, E., Frøseth, M. W., \&Sandberg, N. (2011). Reaching for the unreachable: Identifying factors predicting early school leaving and non-completion in Norwegia nupper secondary education. Scandin avían Journal of Educación al Resecar, 55(1), 225-253.

Narváez, G.A. (2013). La baja tasa de graduación en la Educación Superior Pública argentina. Revista electrónica Gestión Universitaria, 5(3), Recuperado de: http://www.gestuniv.com.ar/ gu_15/v5n3a2.htm.
Quintero, R. y otros cuatro autores. Informe sobre prueba académica y deserción estudiantil en la Universidad Distrital Francisco José de Caldas (2010). http://virtual.udistrital.edu.co/n. pdf Acceso 5 de agosto, (2014) 28. Raman, M. (2013). Factores asociados al abandono y la deserción escolar en América latina: una mirada en conjunto. Revista iberoamericana sobre calidad eficacia y cambio educativo, 11(2), 1-23.

Rinne, R. y T. Järvinen, Dropout and completion in upper secondary education in Finland, School dropout and completion: International comparative studies in theory and policy: 1 (2), 215-232 (2011).

Román, M.C. (2013), Factores asociados al abandono y la deserción Escolar en américa latina: una mirada en Conjunto, Revista Iberoamericana sobre Calidad, Eficacia y Cambio en Educación, 11 (2), 33-59. 